\title{
Hop-by-hop option based flow- handling compared to other IP over ATM protocols
}

Loukola, M. V. and Skyttä, J. O. Helsinki University of Technology Department of Electrical and Communications Engineering P.O. Box 3000, 02015 HUT, Finland phone: +358-9-4512476, fax: +358-9-460224 e-mail:Mika.Loukola@hut.fi

\begin{abstract}
This paper introduces new ways to establish data link level forwarding for IPv6 packets on ATM links. The designed method is merged into to the core of IPv6 protocol. This improves the performance compared to the other traffic-based IP over ATM protocols. (RFC-1953) (RFC-2129)
\end{abstract}

\section{Keywords}

Flow-handling, IP over ATM, IP switching

Performance of Information and Communication Systems U. Körmer \& A. Nilsson (Eds.) (C) 1998 IFIP. Published by Chapman \& Hall 


\section{INTRODUCTION}

From Internet Protocol, Version 6, Specification (RFC-1883) page 28:

A flow is a sequence of packets sent from a particular source to a particular (unicast or multicast) destination for which the source desires special handling by the intervening routers. The nature of that special handling might be conveyed to the routers by a control protocol, such as a resource reservation protocol, or by information within the flow's packets themselves, e.g., in a hop-by-hop option.

From IPv6 The New Internet Protocol (Huitema, 1996) page 130:

Some researchers believe that they can define the quality of service requirements of a given flow in a hop by hop option. This option would be transmitted in some packets. The routers would remember the associated parameters and associate them with the flow.

The paper will study the implementation of the flow-handling based on the IPv6 Hop-by-Hop Options extension header.

\section{LAYER 2 VERSUS LAYER 3 FORWARDING}

Layer 2 forwarding provides simple and fast packet forwarding capability. One primary reason for the simplicity of layer 2 forwarding comes from its short, fixed length labels. A node forwarding at layer 3 must parse a relatively large header, and perform a longest-prefix match to determine a forwarding path. (Doolan, 1996) (RFC-1987)

When a node performs layer 2 forwarding it can do direct index lookup into its forwarding table with the short header. It is arguably simpler to build layer 2 forwarding hardware that it is to build layer 3 forwarding hardware because the layer 2 forwarding function is less complex. (Callon , 1997) (RFC-1954)

By bypassing the conventional IP forwarding (the packet assembly/reassembly) process using cell-relaying, we could dramatically reduce both the IP packet processing delay and the queuing delay at the router. (Esaki, 1997)

Pushing traffic to layer 3 may cause congestion. If data is discarded (Hop Limit = 0 ) or lost (buffer full) TCP will backoff. (RFC-1885) 


\section{LABEL ALLOCATION}

Label allocation can be made both by the downstream node and the upstream node. Downstream allocation is similar to the other designs in the way that the upstream node asks the downstream node to allocate a label for a specific flow. Only in this design no extra request packets are sent. The request for label is carried within every IPv6 packet that belongs to the specified flow. The request for label resides in packet's Hop-by-Hop Option header. Only the label itself needs to be transferred in its own IPv6 packet from the downstream node to the upstream node.

The label resides in the Destination Options header of the IPv6 packet with zero length payload.

The upstream label allocation is very simple. The upstream node can pick up a label and start to send packets belonging to the specific flow using the label. The downstream neighbor needs to reassemble the IPv6 packet in order to make the label bindings to its Label Base (LB). In an ATM switch, the VPI/VCI bindings are link specific so both the upstream and the downstream node know what labels are used and what are unused. The VPI/PCI values are only unique in the physical interface as illustrated in Figure 1. (Esaki, 1997)

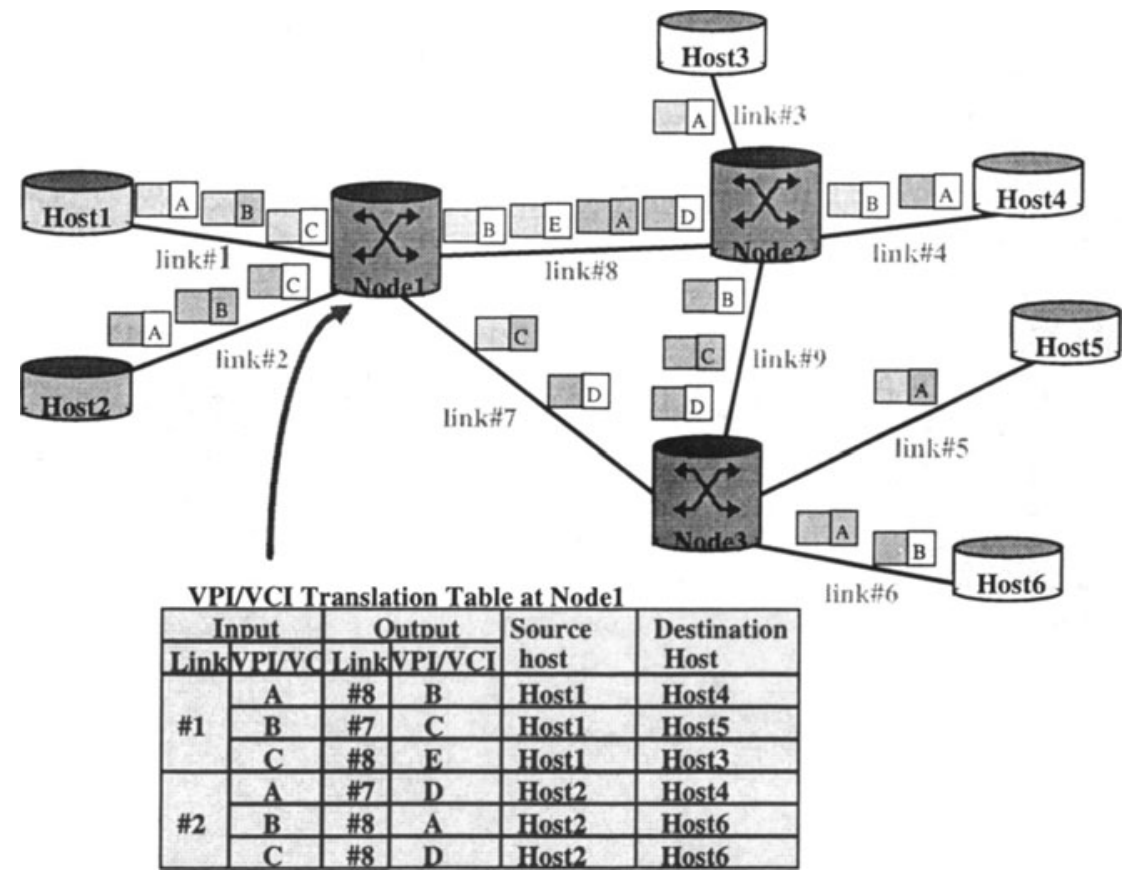

Figure 1. ATM cell multiplexing and relaying 
The upstream label allocation requires some specific features from the ATM switch. It is contingent on ATM switches to keep the cells of a PDU contiguous and in sequence. That is why there is a need for a specific solution in case of upstream label allocation.

\subsection{Downstream label allocation method}

Trigger IP packet starts the cut-through operation (1), Figure 2. The trigger packet has a Hop-by-Hop Options header in its header chain with the Option Type 00110110 (bin). This Option Type is used for all Augmented IP Router Protocol (AIRP) messages. This is just some unique Option Type to be used. This protocol is part of the design and is needed to carry messages between the upstream and downstream node. The trigger packet carries a request for layer 2 forwarding label or layer 3 IPv6 Flow Label for accelerated layer 3 forwarding. Once the $2^{\text {nd }}$ router receives such a request it sends the label to its upstream neighbor in a IPv6 packet (2), Figure 2. This packet has also a Options header with the Option Type 00110110 (bin). This Option header resides in Destination Options header and contains the label to be used for the specific flow. Once the upstream router has received this packet it can start to send packets belonging to that flow labelled with the specific label (3), Figure 3.

When the 3rd router receives the trigger IP packet (4), it sends the label to its upstream neighbor router (5), Figure 3 . The 2 nd Router can now start to send packets belonging to that flow on the dedicated-VC (6). The label allocation process is illustrated in Figures 2-4.

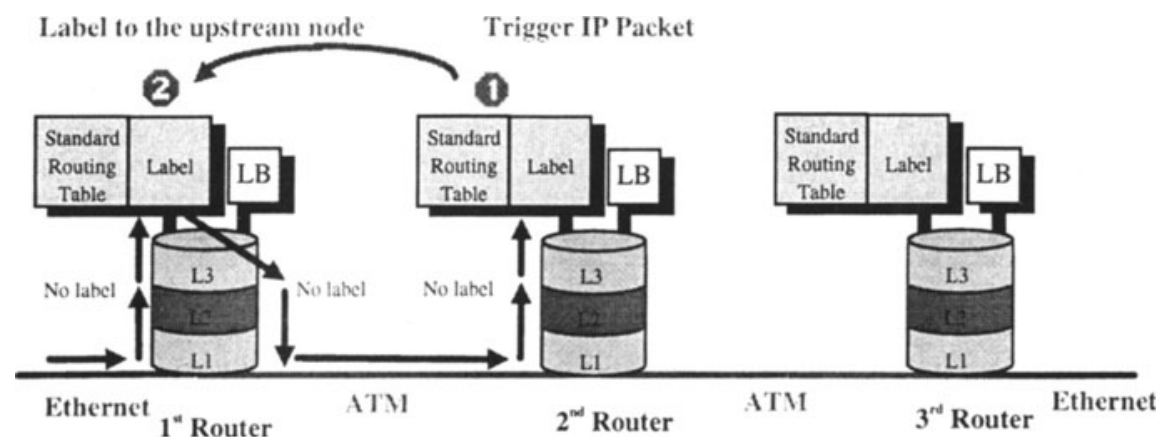

Figure 2. Downstream label allocation \#1 


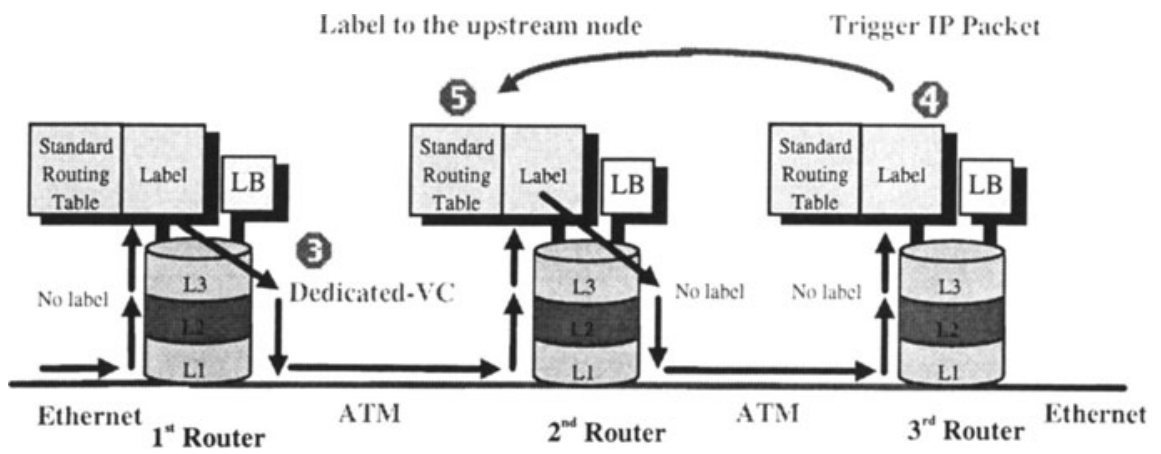

Figure 3. Downstream label allocation \#2

\section{Cut-Through}

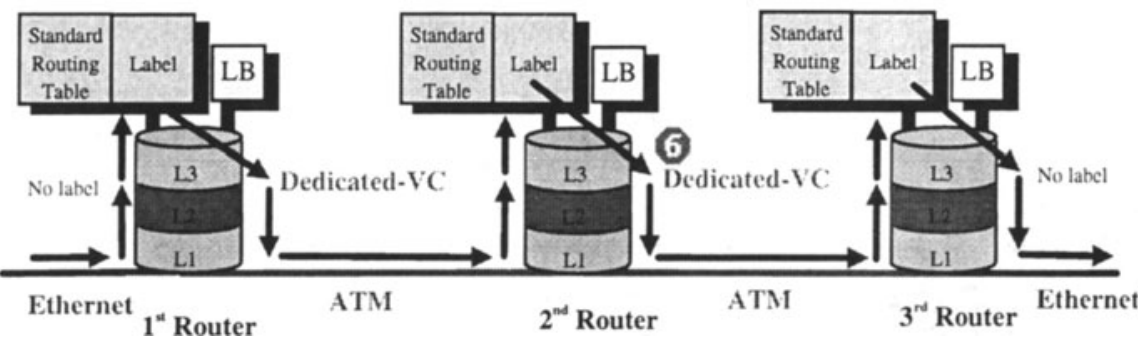

Figure 4. Downstream label allocation \#3

\subsection{Upstream label allocation method}

Another way to achieve cut-through operation is to use upstream label allocation. This means that the upstream node chooses the label (=VCI) to be used. In this case the node choosing the label and the node which needs to interpret packets using that label are not the same node.

When the 1st Router receives trigger IP packet belonging to a new flow, it allocates a label i.e. VCI to it. After that the upstream node is free to send the packets belonging to that flow on the dedicated-VC (1). When the downstream node receives cells on the new VCI that it has no entry in its LB, it has to reassemble the IPv6 packet is order to determine where the packet is going (2). After the packet is reassembled, a next link label is allocated and a entry is entered to the LB. After that the 2 nd router begins to send cells to the next router on this new dedicated-VC (3). After the cells belonging to the flow arrive at the border node of the ATM domain (3rd router), the packet is once again reassembled and sent on the non-ATM link (4). The cut-through establishment is illustrated in 
Figure 5. The following IP packets receive the cut-through treatment at the 2 nd router (5) as shown in Figure 6.

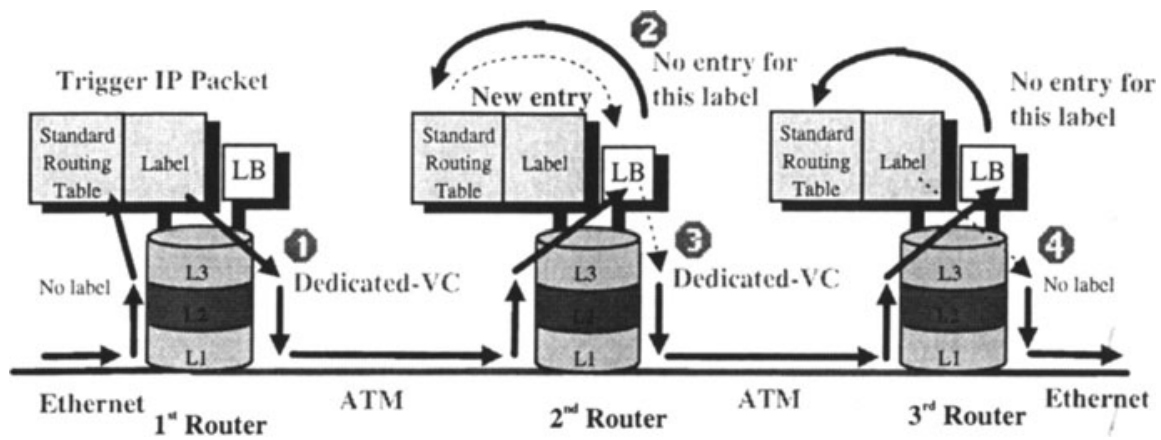

Figure 5. Upstream label allocation \#1

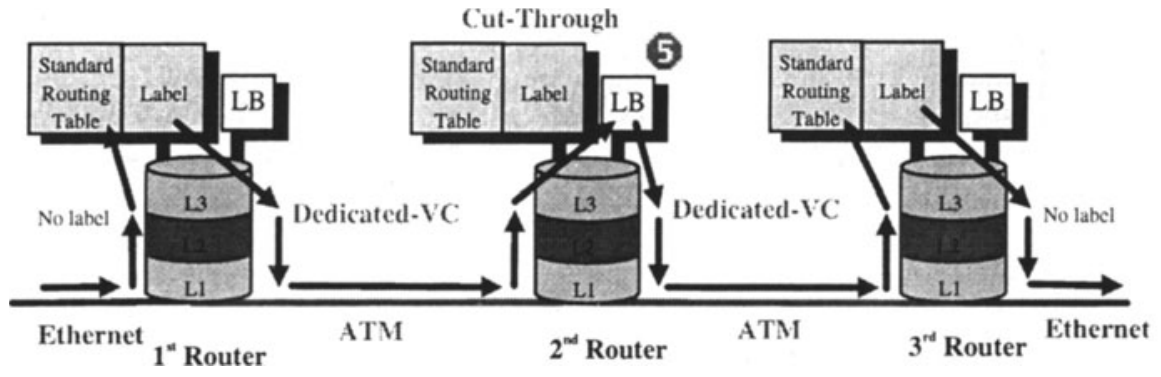

Figure 6. Upstream label allocation \#2

\section{LABEL DISTRIBUTION}

Label distribution occurs between ATM switches which have been augmented with standard IP routing support. The IP routers must be able to recognize the IPv6 Option type (00110110 bin) used in this design. Such IP routers are referred as Augmented IP Routers (AIRs). The word augmented here refers to the AIRs ability to recognize the needed IPv6 Option type.

In the downstream label allocation mode the request for label is passed in the IPv6 Hop-by-Hop Options header and the label is passed to the upstream node in the IPv6 Destination Options header. Both the Destination Options and the Hop-byHop Options headers can contain Options in the same format. (RFC-1883) 
Labels expire when there has not been any packet carrying that specific label for 180 seconds.

\subsection{Traffic Based Soft-State Label Base Bindings}

The AIRP protocol exchanges layer 2 labels based on traffic like IP switching. (RFC-1953) This reduces the overhead of exchanging labels between all peers in a routing domain and reduces the size of the label binding information bases in routers.

Topology based methods have the ability of forwarding all the packets on layer 2 including the first packets of each flow, while in traffic based methods the first packet has to be reassembled in all the routers along the packets delivery path. But this was not a sufficient argument to make AIRP a topology based protocol. Simplicity is beautiful.

The nature of the bindings in the LB is soft-state because the connection is established due to the request in the first IPv6 packet. But on the other hand there is no refreshment procedure or keep-alive messages between the neighboring Augmented IP Router (AIRs).

\section{AUGMENTED IP ROUTER PROTOCOL}

The AIRs need to exchange information with each other. That is why a simple Augmented IP Router Protocol (AIRP) is needed. The messages are transferred within the IPv6 packet's Hop-by-Hop Options header or the Destination Options header. (RFC-1883, RFC-1884)

\subsection{Message Types}

Three kind of message types are defined: 1) request for label message, 2) label transfer message, and 3) label removal message.

\section{Request For Label Message}

This message must be within all the IPv6 packets belonging to the same flow that want special AIRP treatment. The first packet triggers the downstream label allocation procedure. Table 1 shows the format of the Hop-by-Hop Options header. 
Table 1 Format of the Request For Label message

\begin{tabular}{lll} 
octet & field data \\
\hline $1^{\text {st }}$ & Next Header & \\
$2^{\text {nd }}$ & Hdr Ext Len & 00000000 \\
$3^{\text {rd }}$ & Option Type & 00110110 \\
$4^{\text {th }}$ & Opt Data Len & 00000100 \\
$5^{\text {th }}$ & Action & 00000001 \\
$6^{\text {th }}$ & Reserved & 00000000 \\
$7^{\text {th }}$ & Reserved & 00000000 \\
$8^{\text {th }}$ & Reserved & 00000000 \\
\hline
\end{tabular}

After a downstream AIR receives this message it allocates a 24-bit label to be used for the flow, and enters that label to its LB. After the label is entered to LB, the downstream AIR sends a label transfer message to the upstream AIR.

\section{Label Transfer Message}

This message is a response to the label request message. Table 2 shows the format of the Destination Options header.

Table 2 Format of the Label Transfer Message

octet

field data

$\begin{array}{ll}1^{\text {st }} & \text { Next Header } \\ 2^{\text {nd }} & \text { Hdr Ext Len }=00000101 \\ 3^{\text {rd }} & \text { Option Type }=00110110 \\ 4^{\text {th }} & \text { Opt Data Len }=00101100 \\ 5^{\text {th }} & \text { Action }=00000010 \\ 6^{\text {th }}-8^{\text {th }} & \text { Label } \\ 9^{\text {th }}-24^{\text {th }} & \text { Source Address of the IP packet that triggered downstream a. } \\ 25^{\text {th }}-40^{\text {th }} & \text { Destination Address of the IP packet that triggered downstream a. } \\ 41^{\text {st }}-43^{\text {rd }} & \text { Flow Label of the IP packet that triggered downstream a. } \\ 44^{\text {th }}-48^{\text {th }} & \text { Reserved }\end{array}$

As the upstream AIR receives this message, it is ready to use the VCI for the specified flow. 


\section{Label Removal Message}

If the upstream node wants the downstream node to delete a LB binding it can send a label removal message instead of waiting the 180 seconds. This is desired if the number of ongoing flows is near to the maximum, but otherwise it is a waste of bandwidth. The format of this message is shown in Table 3.

Table 3 Format of the Label Removal Message

\begin{tabular}{ll}
$1^{\text {st }}$ & Next Header \\
$2^{\text {nd }}$ & Hdr Ext Len $=00000000$ \\
$3^{\text {rd }}$ & Option Type $=00110110$ \\
$4^{\text {th }}$ & Opt Data Len $=00000100$ \\
$5^{\text {th }}$ & Action $=00000011$ \\
$6^{\text {th }}-8^{\text {th }}$ & Label \\
\hline
\end{tabular}

This message is sent on default VCI. After this the downstream node deletes the binding from its LB and can make use of the same label immediately after the deletion.

\section{SIMULATIONS}

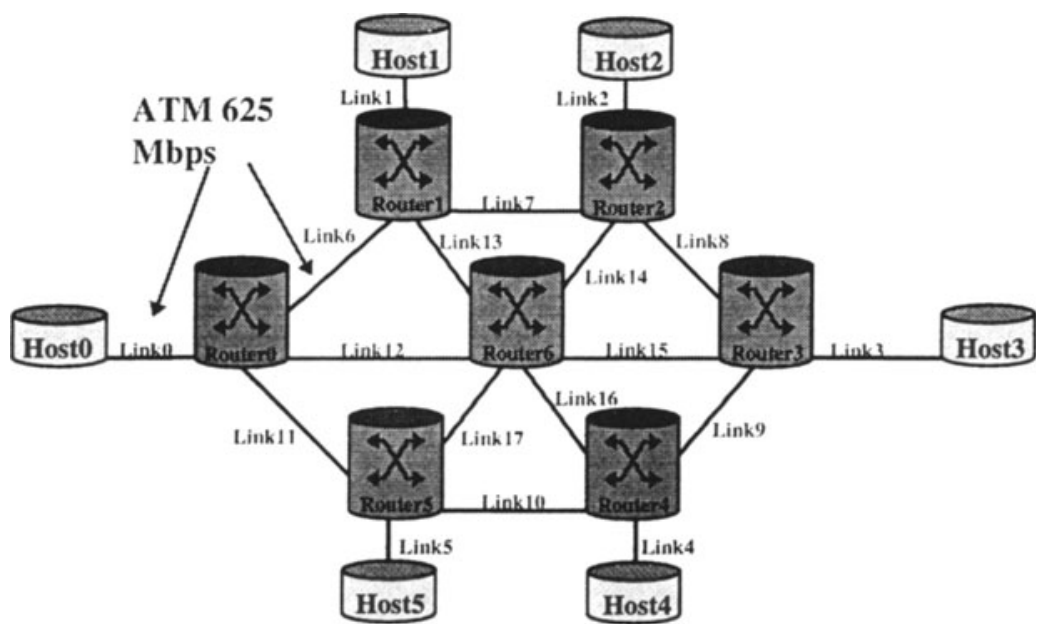

Figure 7. The simulation platform 
The IP over ATM protocols were simulated in a fixed platform illustrated in Figure 7. The simulator is based on the performance of real ATM switches available from Cisco Systems. The throughput of layer 2 forwarding (1.357 Gbps) is taken from the LightStream 2020 Multiservice ATM Switch specifications (Cisco, 1995). The throughput of layer 3 forwarding is taken from the TCP/IP Router Tests, v2.5 (Digital, 1997). The throughput depends on the packet size. The average layer 3 forwarding speed is 7.25 Mbps when packet sizes of 64, 128, 256, $512,1024,1518$ bytes are used randomly. (Loukola, 1997)

In the simulation each host has ten ongoing flows to other hosts. The destination of each flow is chosen randomly. The average flow lengths can be configured. When one flow is ended another is established. At all times each host has ten ongoing flows. Each individual packet of each flow is randomly picked from the six possible packet sizes listed above. This simulation platform gives the possibility to find the maximum forwarding speed of Router 6 that is the bottleneck of the network. When the number of IP packets in the layer 3 queue of a router exceeds the buffer size the load has been too heavy. The maximum forwarding speed of the Router 6 can be found by adjusting to transmitting speeds of the hosts. When the peak layer 3 queue size of the Router6 is between $90 \%$ and $100 \%$, the forwarding speed can be considered the maximum one.

The optimistic estimation of the effect of cached flow-handling (CFH) in layer 3 performance is used. An existing CFH entry in a router is estimated to decrease the layer 3 processing delay to 5 percent of the normal processing time.
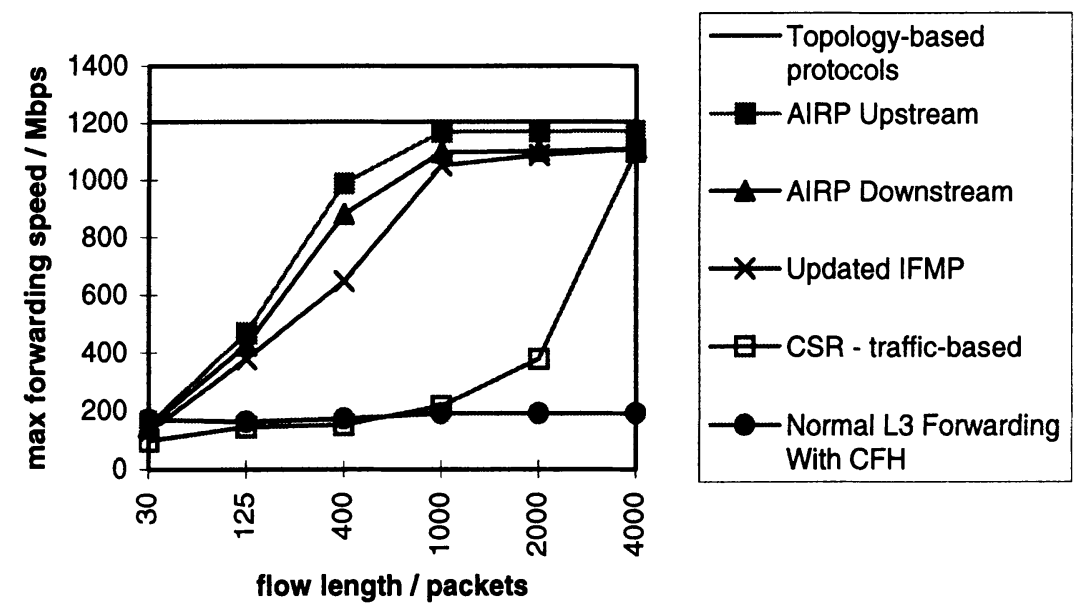

Figure 8. Simulation Results

Topology-based protocols like Tag Switching, Aggregate Route-Based IP Switching (ARIS), and Switching IP Through ATM (SITA) have the maximum 
forwarding speed in spite of flow length because the ATM switch is configured prior to the IP packet transmissions. AIRP with its upstream label allocation proves its power. AIRP is merged to the core of IPv6. This reduces processing overhead in the routers. (Feldman, 1997) (Viswanathan, 1997) (Cisco, 1997) (Heinänen, 1996) (Davie, 1997)

\section{REFERENCES}

Feldman, N. and Viswanathan, A. (1997) ARIS Specification, Internet Draft <draft-feldman-aris-spec-00.txt>, IBM Corporation, March 1997

Viswanathan, A., et. al. (1997) ARIS: Aggregate Route-Based IP Switching, work in progress, Internet Draft <draft-viswanathan-aris-overview-00.txt>, IBM Corporation, March 1997

Loukola, M. (1997) Data Link Level Forwarding for IPv6 Packets On ATM Links, Licentiate's Thesis, TKK, November 1997

Callon, R., et. al. (1997) A Framework for Multiprotocol Label Switching, Network Working Group, Internet Draft <draft-ietf-mpls-framework-00.txt>, May 1997

Conta, A. and Deering, S. (1995) Internet Control Message Protocol (ICMPv6) for the Internet Protocol Version 6 (IPv6) Specification, RFC 1885, Digital Equipment Corporation, Xerox PARC, December 1995

Deering, S. and Hinden, R. (1995) Internet Protocol, Version 6, Specification, RFC 1883, Xerox PARC, Ipsilon Networks Inc., December 1995

Hinden, R. and Deering, S. (1995) IP Version 6 Addressing Architecture, RFC 1884, Ipsilon Networks Inc., Xerox PARC, December 1995

Newman, P., et. al. (1996) Ipsilon Flow Management Protocol Specification for IPv4 Version 1.0, Ipsilon Networks Inc., RFC 1953, May 1996

Newman, P., et. al. (1996) Ipsilon's General Switch Management Protocol Specification Version 1.1, Ipsilon Networks Inc., RFC 1987, August 1996

Huitema, C. (1996) IPv6 The New Internet Protocol, Prentice Hall PTR, 1996

Cisco Systems (1997) LightStream 2020 Multiservice ATM Switch", Cisco Brochure, Cisco Systems Inc., September 1995

Cisco Systems (1997) Scaling the Internet With Tag Switching, White Paper, url: http://www.cisco.com/warp/public/732/tag/pjtag_wp.htm, url valid: october 20, 1997, Cisco Systems Inc., April 1997

Doolan, P., et. al. (1996) Tag Distribution Protocol, work in progress, Internet Draft <draft-doolan-tdp-spec-00.txt>, Cisco Systems, Inc., September 1996

Digital Equipment Corporation (1997) TCP/IP Router Tests, v2.5, Digital Equipment Corporation, July 1997

Nagami, K., et. al. (1997) Toshiba's Flow Attribute Notification Protocol (FANP) Specification, RFC 2129, Toshiba Corporation, April 1997 
Newman, P., et. al. (1996) Transmission of Flow Labelled IPv4 on ATM Data Links Ipsilon Version 1.0, Ipsilon Networks Inc., RFC 1954, May 1996 Heinänen, J. (1996) Updated SITA Proposal, Telecom Finland, November 1996 Davie, B., et. al. (1997) Use of Tag Switching With ATM, Internet Draft <draftdavie-tag-switching-atm-01.txt>, Cisco Systems, Inc., January 1997

Esaki, H., et. al. (1997) White Paper on CSR (Cell Switch Router) Provided by Toshiba Corporation, Toshiba Corporation, April 1997

\section{BIOGRAPHY}

Mika Loukola works currently at Helsinki University of Technology as a research engineer in the Laboratory of Signal Processing and Computer Technology. Loukola has recently graduated as a Licentiate of Technology. His Licentiate's Thesis Data Link Level Forwarding for IPv6 Packets On ATM Links studied the wide spectrum of IP over ATM protocols. His previous Master's Thesis ATM Network Emulator for Private Network-to-Network Interface studied the ATM Forum specifications for high speed data communication. Loukola is currently involved with priority based IP networking.

Jorma Skyttä is associate professor of signal processing and computer technology at Helsinki University of Technology. He holds Doctor of Technology from the same university at the area of digital signal processing implementations. His current research interests include high speed data communications. 